\title{
2. IRREGULAR VARIABLE STARS OF THE RW AURIGAE TYPE
}

\author{
P. N. KHOLOPOV \\ Astronomical Council, Academy of Sciences, Moscow, U.S.S.R.
}

The RW Aurigae type was first introduced by P. P. Parenago [1] in $193^{2}$ for the designation of irregular variable stars that belong to spectral class $G$ and show extremely rapid (up to I magnitude in several hours) and large (up to 3 or 4 magnitudes) variations of their brightness. Many stars of that type were found to be connected with dark diffuse nebulae. Some of them, as for instance R Monocerotis, RY Tauri, and R Coronae Australis, were also associated with bright and dark comet- or fan-shaped nebulae, often themselves variable.

There existed a double terminology for the designation of the irregular variables connected with the diffuse nebulae. Such stars are often named the Orion Nebula type variables, because a particularly numerous group of irregular variables that consists, according to Parenago, of not less than 220 members [2] is connected with this Nebula. The term 'RW Aurigae type variables' also is used for the designation of isolated variable stars of the above-mentioned properties. In the course of time it has frequently happened that the same stars have been referred by some observers to the RW Aurigae type, and by others to the Orion Nebula type, owing to their striking connexion with the dark diffuse matter.

The paper by Joy on ' $T$ Tauri Variable Stars' appeared in $1945^{\text {[3]. The }}$ following features should be present, according to Joy, in order to assign a star to this new type: (a) irregular variations of brightness with an amplitude of about 3 magnitudes, $(b)$ an $\mathrm{F}_{5}$ to $\mathrm{G}_{5}$ type spectrum with $\mathrm{H} \alpha$ and $\mathrm{Ca}$ II emissions, as in the spectrum of the solar chromosphere, $(c)$ low luminosity, and $(d)$ connexion with a dark or bright nebula.

Joy revealed, in fact, one of the most important properties of the RW Aurigae type variables: the existence of a typical emission spectrum, which had escaped the attention of earlier observers. At the same time, he introduced the term ' $T$ Tauri type variable', which soon became popular along with the designation ' $\mathrm{RW}$ Aurigae type'.

In 1949 Hoffmeister, in his paper on 'RW Aurigae Type Stars and their Related Types', [4] described the results of his studies of the light 
variations of these variables. The chief criterion for referring stars to the type, which Hoffmeister proposed to call the RW Aurigae type in an extended sense, is solely, in his opinion, the general outline of the light curve. Hoffmeister believes that an essential feature of this type of variable is the existence of rapid, non-periodic fluctuations, interrupted by intervals of rest (the novae and U Geminorum type stars are excluded). The replacement of intervals of activity by periods of weak or slow variation, or even of constant brightness, characterizes the RW Aurigae type stars. In general, variations of 0.5 to I magnitude per day, or sometimes per hour, are considered to be rapid. Such variations are usually not observed in the case of slow irregular variables which are red giants. Rapid variations are often superimposed upon slow variations of the mean brightness.

The term 'in an extended sense' refers to the fact that among the variables resembling RW Aurigae in the character of their light variation, stars are met not only of class $\mathrm{G}$, but also of all the other spectral classes from $B$ to $M$. The distances of the dark nebulae, in the directions of which the majority of variables of that type are concentrated, and the spectral properties established for some of these stars, testify that the absolute magnitude of the variables approximate those of main sequence stars of corresponding spectral classes.

In $1946 \mathrm{Joy}[5]$ discovered forty faint stars having the $\mathrm{H} \alpha$ line bright that were located in the regions of dark nebulae in Taurus, Auriga, and Orion. Their emission spectra appeared to be extremely similar to the spectra of $T$ Tauri type variables, described by him earlier; they were mainly dwarfs of spectral classes A-M [6].

In $195 \mathrm{I}$ it was discovered by me, together with N. E. Kurochkin, that almost all of these stars were also variables that belonged, from their spectral properties, to the RW Aurigae type [7].

At about the same time clusterings of stars having bright $\mathrm{H} \alpha$ were discovered in a number of other diffuse nebulae by Struve and Rudkjøbing [8], Haro and Moreno [9], and also by .Herbig [10]. Many of these stars were found to be variables. Emission lines were also found in the spectra of a number of irregular variables in the nebulae of Orion [11,12] and NGG 2264 [13]. Further study of the spectra of these variables [14, 15] showed their similarity to the spectra of the RW Aurigae type variables that are connected with the dark clouds in Taurus and Auriga.

Thus, there are no grounds at present to retain the term 'stars of the type of the Orion Nebula variables' along with the term ' $R W$ Aurigae type variables', in so far as there is no essential difference between these two notions. 
Several attempts to classify the RW Aurigae type variables have been made. Hoffmeister's classification [4] is based only upon the outline of the light curve. This is the reason for its restriction. The classification by Parenago [16] is, to some extent, similar to Hoffmeister's. It was suggested by Parenago in the course of his study of -irregular variables in the Orion Nebula, and is based upon the frequency distribution of magnitudes of a given variable. It may be supposed, however, that the distribution of magnitudes can only be used to describe the behaviour of an irregular variable in a given epoch, but not for its classification.

At the same time, among the most typical representatives of the T Tauri type (as, for instance, T Tauri and RW Aurigae), variables with both rapid and slow variations of their brightness are met. It may, therefore, be believed that the rate of light variation does not characterize the type, but rather the state of the star, and perhaps also its age.

The most advisable classification-according to physical propertieshas not yet been developed. There are grounds for its creation, however, that are afforded by the studies of Joy and Herbig, who suggested schemes for the classification of the emission stars connected with the diffuse nebulae.

Similarly to Hoffmeister, who introduced the RW Aurigae type in an extended sense, Herbig has practically introduced the $T$ Tauri type in an extended sense, as well. He assigned to that type not only the Ge stars, but also stars of much later types, up to $\mathrm{M}$, inclusively. The difference between the two systems is that Herbig bases the type upon the spectral featuresin particular on the emission spectrum and not upon the photometric characteristics.

In the case of Hoffmeister's classification, the spectral class and luminosity are not essential data. In Herbig's classification, contrary-wise, the character of light variations is not essential, in so far as it is assumed that the majority of (or perhaps all) stars with emission spectra of the T Tauri type are variable.

The question about the correspondence of the two types arises immediately. Do all the stars referred to the RW Aurigae type according to their photometric features possess spectral characteristics inherent to the $T$ Tauri type in Herbig's sense, and vice versa? Such a question can at once be answered negatively. Thus, for instance, $\mathrm{R}$ Monocerotis has a spectrum typical of the $\mathrm{T}$ Tauri type stars, but has never shown any rapid light variations and is not referred by Hoffmeister to the RW Aurigae type. T Tauri itself is a similar case. We refer these stars to the RW Aurigae type on the basis of their spectral features. On the other hand, Herbig 
showed [17] that the majority of variables that have been referred to the RW Aurigae type on the basis of their photometric behaviour alone, and which are not, as a rule, members of systems associated with diffuse nebulae, do not have emission spectra.

Herbig concludes that the RW Aurigae type variables in Hoffmeister's sense do not form a physically homogeneous group. He doubts even the physical reality of this type. His conclusion is based on the fact that the spectral types of a number of RW Aurigae type stars are contained in the limits from $\mathrm{B}_{5}$ to $\mathrm{M}_{4}$, while their luminosity classes range from $\mathrm{Ib}$ (super-giants) to V (dwarfs). In Herbig's opinion, it is not easy to find a common cause of the light variations that could act in objects as different as are the RW Aurigae type stars in Hoffmeister's sense.

However, such a cause could be processes arising from the non-stability of these stars. It is possible that all these objects, regardless of their spectral classes and luminosities, are of recent origin and that their luminosities are not yet quite stabilized.

It is known that a number of RW Aurigae type stars in the Orion and NGG 2264 nebulae have no bright lines in their spectra. On the other hand, as was established by Herbig and Haro, the intensity of $\mathrm{H} \alpha$ emission is often variable for such stars and may completely disappear in their spectra.

Thus the RW Aurigae type consists both of emission and non-emission variables, of extremely diverse physical properties. The question of the homogeneity of the type and the similarity or difference of the causes of light variation of the stars related to that type is very complicated, however, and various possibilities must be taken into account in its solution.

In connexion with the actual variety of the physical properties of the RW Aurigae type variables it is extremely important to have a criterion that permits one to judge with some certainty whether a star belongs to that type. This criterion can be sought in the fact that the cause of the light variability of the examined stars is the same.

The main argument supporting a unique cause of the light variation of the majority of RW Aurigae type variables lies in the fact that these variables are, as a rule, found together, in groups which we now call Tassociations. This term was first introduced by V. A. Ambartsumian [18], who noticed that of eleven bright $\mathrm{T}$ Tauri type variables studied by Joy, seven are located in the same area of the sky, in the region of Taurus and Auriga. Some groups of the RW Aurigae type variables as, for instance, in the Orion Nebula, around $\mathrm{R}$ Coronae Australis, and elsewhere, were recognized a long time ago. At present, twenty-three $\mathrm{T}$-associations are 
known to us [19]. It must be noted that T-associations are found in the form of nuclei in $\mathrm{O}$-associations. They are known for about all of the nearest $\mathrm{O}$-associations. At the same time, doubtlessly, there exist T-associations that do not enter into the composition of $\mathrm{O}$-associations and do not contain O or Bo type stars.

What is the cause of the light variation of the RW Aurigae type stars? For several decades, it was considered that the RW Aurigae type stars are 'obscured' variables. Later on, when it was found that the light variations of many such stars are accompanied by changes of their spectral classes, it became clear that the cause of the light variations should be sought in physical processes taking place in the upper layers of the stars. The Orion Nebula variables, which were for a long time the classical examples of the obscured variables, had to be excluded from that category.

This does not mean, of course, that obscured variables do not exist at all. No convincing example may be given at the present time, however, where the light variation is caused by a change of the optical thickness of the diffuse matter lying between the star and the observer.

If the change of the conditions of obscuration plays a part in the light variations of the above-mentioned emission variables, this part is not an essential one. It should, however, be emphasized that there are no indisputable proofs of the assumption that the nature of the non-emission and emission stars of the RW Aurigae type is similar. The assumption of their similarity is supported by the variability of the emission spectra and the similarity of their distributions in the nebulae. Strictly speaking, the arguments against the hypothesis of obscuration are based upon the fact that a quite improbable structure of the dark nebula is required in order to explain the observed rapid light variations of these stars. That is, extremely large local density gradients or small condensations are demanded. But if it were assumed that $\mathrm{T}$-associations are regions of star formation, then it cannot be denied that the structure and properties of the nebulae connected with $\mathrm{T}$-associations might really be of an extraordinary character. Although it seems to us that the non-emission variables represent the subsequent stage of the evolution of emission variables, the role of obscuration in the light variation of the non-emission RW Aurigae type stars cannot be altogether rejected at present.

The variability of the emission spectrum accompanying the light variation of the emission stars cannot, of course, be explained by obscuration. It is caused by some other more complicated physical processes.

There are at present two points of view regarding the nature of these processes. It is considered, on the one hand, that the RW Aurigae type 
variables are ordinary stars which entered the dark cloud at random and are changing their brightnesses owing to processes of interaction with the surrounding diffuse matter. It is sufficient to eliminate, from this point of view, the dark cloud, and the emission lines in the spectrum of the star will disappear, and the changes of brightness will stop. On the other hand, it is supposed that the cause of variability lies in the stars themselves, in which case these stars are not ordinary ones.

Investigators of the spectra point out the difficulties in the spectral classification of these objects, the absorption lines often being veiled by strong continuous emission. This emission continues far into the violet. The distribution of energy in the continuum is essentially different from that in the continuous spectrum of an ordinary cool dwarf.

In order to explain the appearance of the emission lines in the spectra of stars connected with nebulae and the light variation of such stars, Greenstein [20] advanced a hypothesis that the cause of such phenomena is the same as in the case of flares in the solar chromosphere according to Giovanelli's theory. In that theory, the electrons are accelerated in the variable magnetic field of sunspots, and atoms are excited through electron collisions. According to Greenstein's opinion, the streams of ionized dark matter falling upon the star create variable magnetic fields of such intensity that the spectrum of the flares becomes visible in the general spectrum of the star.

We admit that hypotheses similar to that of Greenstein may possibly explain the appearance of a faint emission spectrum in the case of ordinary dwarfs which enter the dark cloud at random. Such emission may become visible if the processes of the interaction of the star with the surrounding diffuse matter become sufficiently important. We do not know, however, the extent to which these effects are appreciable. It should be extremely important to establish with certainty the spectroscopic effects caused by such interaction, in order to be able to separate them from the properties inherent to the spectrum of the star itself.

Haro, Iriarte and Chavira [21] called attention to the fact that stars showing strong $\mathrm{Ca}$ II, but weak $\mathrm{H} \alpha$ emission, and belonging to the $\mathrm{CO}$ Orionis association, are located in the $\mathrm{H}$ in region embracing the system of $\lambda$ Orionis. In the region of dark nebulae in Taurus and Auriga, where there is, apparently, much neutral hydrogen, bright $\mathrm{H} \alpha$ lines in the spectra of emission stars are extremely intense. Haro, Iriarte and Chavira believe that this indicates that the spectral composition of radiation of the emission stardepends upon the state and nature of the surrounding diffuse matter. We do not see, however, any causal relationship between the above phenomena. 
Returning to the hypothesis by Greenstein, we have to point out that it is extremely difficult to admit that the light variation of from $I$ to 3 magnitudes observed in RW Aurigae type variables might be explained by means of this hypothesis. It is doubtful if a source of emission not connected with the star might, under its influence, emit an amount of energy exceeding the energy of radiation of the star itself by two or three, or even ten times.

It must be taken into account that the production of the phenomena observed in the spectra of the RW Aurigae type stars can take place without the participation of diffuse matter. This is proven by the fact that in the spectra of UV Ceti type stars (variable dwarfs located in the vicinity of the Sun, with spectral classes from $\mathrm{dM}_{3} \mathrm{e}$ to $\mathrm{dM}_{\mathrm{M}} \mathrm{e}$ ) similar emissions are observed during the outbursts, and $\mathrm{H} \alpha$ and $\mathrm{Ca}$ II emission lines are observed in them permanently, between the flares.

Open clusters containing G-type stars (as, for instance, the Pleiades), with which dust clouds are connected, are known. The density of these clouds can hardly be less than the density of the peripheral parts of the nebula in which such a typical emission and variable star as RW Aurigae itself is located. In the Pleiades, the stars of which represent excellent photometric standards, no faint stars similar to RW Aurigae are found. They should have been discovered a long time ago if they were present.

This permits one to suggest that the emission and variability of the RW Aurigae type stars is caused not by the diffuse matter, but by the stars themselves.

It is of great interest to recall the hypothesis suggested by V. A. Ambartsumian [22] and I. M. Gordon [23], to the effect that the continuous emission observed in the spectra of the RW Aurigae type variables and UV Ceti type stars during their outbursts is not of a thermal character. These authors explain this phenomenon in terms of the radiation emitted by relativistic electrons in the hypothetical magnetic fields of these stars. Ambartsumian suggested also another explanation of that phenomenon, involving the direct release of energy by stellar matter from the deep interior that is ejected into the external atmospheric layers of these stars.

RW Aurigae type stars cannot be ordinary stars that have. entered the diffuse nebulae at random, as otherwise the fact of their concentration into small volumes of comparatively large diffuse nebulae cannot be understood. Greenstein and Herbig showed that the spatial density of stars in these nebulae exceeds considerably the density of ordinary stars of corresponding types in the vicinity of the Sun.

$\mathrm{T}$-associations are actual clusters. Theirformation cannot be explained by the collisions of dwarf stars with nebulae, and the gradual accumulation 
of the stars therein by a process of capture by the nebulae. This mechanism has been described by L. E. Gurevitch [24]. The spectral composition of different $T$-associations, some of which contain mainly one type of stars while others contain stars of a different kind, would otherwise be inexplicable. Thus, for example, there are no G-type stars in the CO Orionis association, but many $\mathrm{K}_{3}-\mathrm{K}_{4}$-type stars. In the UZ Tauri association, at the same time, a number of G-type stars are found, but none of types $\mathrm{K}_{3}-\mathrm{K}_{4}[7]$.

Neither can it be assumed in such cases that a whole cluster of ordinary dwarfs has entered a nebula, because some explanation would then have to be given of the fact that the cluster is found exactly in the centre of the diffuse nebula, as is observed in the Orion Nebula and in NGC 2264. The genetical connexion of these systems of stars with the surrounding nebulae has been suggested by a number of investigators.

The average density of some $\mathrm{T}$-associations is so low that they can hardly be considered dynamically stable systems [7].

In a number of $\mathrm{T}$-associations, nuclei (regions of increased density) are observed. Such nuclei are striking in all of the more or less studied T-associations: those in Taurus, Auriga, Orion and Monoceros. The richest $\mathrm{T}$-association in Orion has four nuclei, located along a straight line with equal intervals between their centres, thus forming a chain. This is the system of Orion's Sword, which contains the famous Trapezium in one of its nuclei. Such a system can by no means be considered a random formation, or an old one. It may be of recent origin, having had as yet no time to disintegrate.

The following facts also testify that stars belonging to the $\mathrm{T}$-associations are unusual.

Herbig [13] discovered that the absorption lines of four typical emission stars (T Tauri, UX Tauri, RY Tauri and SU Aurigae) are anomalously wide and diffuse. This is never observed in the case of ordinary dwarfs of the corresponding spectral classes. According to Herbig's opinion, the phenomenon can be explained as due to either high rotational velocities of these dwarfs, or to strong turbulence in their atmospheres. The choice between these two possibilities cannot yet be made, but in either case this phenomenon deserves much attention.

Let us point out also that Sanford [25] has observed that the spectrum of $\mathrm{T}$ Tauri suggests the probability of ejection of matter from the atmosphere of that star.

The sum of all the above-mentioned facts testifies that the RW Aurigae type stars are, chiefly, stars of recent origin. This point of view was first 
proposed by V. A. Ambartsumian [26]. It is not excluded that among the RW Aurigae type variables are to be found ordinary stars which have entered the dark clouds at random. We are unable as yet to separate such stars from emission stars of constant brightness that actually belong to $\mathrm{T}$-associations. It is not impossible that among the non-emission RW Aurigae type variables, so classified according to their photometric behaviour only, there will be found a large number of stars that are erroneously referred to that type. But all this can hardly affect the conclusions that follow from the study of the properties of the majority of variables of that type, which do belong to T-associations.

Herbig restricts the $T$ Tauri type to stars in the vicinity of the main sequence, with spectral classes of type $G$ and later. But there are no reasons for us to believe that the variability of stars of earlier classes and even of different luminosities (for example, giants or sub-giants) that belong to the $\mathrm{T}$-associations differs by its nature from that of stars of later types.

We assign at present to the RW Aurigae type stars those irregular variables which are located on the spectrum-luminosity diagram in the region of the complete main sequence (from spectral classes $\mathrm{O}$ to $\mathrm{M}$ ), and in the region of the sub-giants. These variables possess emission spectra in the majority of cases and are mainly characterized by rapid variations of brightness. However, among them stars are found in whose spectra emission is present only weakly, sometimes disappearing completely, as well as stars having comparatively slow light variations.

By the T Tauri stars, we understand merely a group of RW Aurigae type stars of spectral class Ge, as was initially suggested by Joy.

It is not excluded, that the luminosities of some typical variables of the $\mathrm{T}$ Tauri class, like $\mathrm{R}$ Monocerotis and $\mathrm{R}$ Coronae Australis (i.e. variables connected with comet-like nebulae), will appear to be significantly higher than the luminosities of main sequence stars of corresponding spectral classes. Some of these variables may even be super-giants. This cannot, however, be a reason to exclude such variables from the category under consideration.

The question regarding the direct connexion of UV Ceti type variables with the RW Aurigae type variables remains open for the present time. Even in the nearest $T$-associations we do not know stars of spectral classes later than $\mathrm{dM}_{2} \cdot 5$. The earliest of all the known UV Ceti type stars belong to spectral class $\mathrm{dM}_{3}$ e. The so-called rapid variables, which were discovered by Haro and Morgan [27] in the Orion Nebula and which are reminiscent of UV Ceti type stars in the character of their light variation, 
have higher luminosities and are, obviously, a variety of the RW Aurigae type variables.

These conclusions permit one to formulate the following working hypothesis for the systematization and interpretation of the results of observation. It may be believed that RW Aurigae type variables are stars of recent origin, which are in process of formation (Ambartsumian proposed the use of the term 'stars in the making' for them), and that they define one of the initial stages of the life of main sequence stars, of subgiants, and possibly also of other types.

This does not mean, however, that stars originate initially in the form of RW Aurigae type variables. Ambartsumian [22] indicated that possibly the form that preceeds the RW Aurigae type stars might be the Herbig-Haro Objects: i.e. the small bright round nebulae with which only very faint blue stars can be associated. Only seven objects of that kind are known in the Orion Nebula region, three of them forming a chain of about 5 ' in length.

We think that RW Aurigae type variables might suddenly originate from a preceding stage. The remarkable star FU Orionis that flared up in the centre of a small dark globule, was found to be connected with a bright fan-shaped nebula. In this case, we actually observed the appearance of the comet-shaped nebula and the star connected with it. It is not excluded that in FU Orionis we are eye-witnesses of the actual formation of an object similar to R Monocerotis or R Coronae Australis, and it is also not impossible that these objects originated like FU Orionis. This star deserves careful watching. It is not impossible that the typical emission spectrum will appear in the future, and slow variations of brightness, similar to that observed in $\mathrm{T}$ Tauri and $\mathrm{R}$ Monocerotis, will set in. If this hypothesis (being as yet on the verge of fantasy) is correct, then it may be supposed that the rapid light variations set in during the latest stages of the evolution of the RW Aurigae type variables, previous to their transition into a stable state. This conclusion is based upon the fact that slowly varying RW Aurigae type variables appear to be strongly connected with diffuse nebulae, whereas stars of that type whose connexion with the diffuse nebulae is less evident, show mainly rapid light variations. 


\section{REFERENGES}

[1] P. P. Parenago, Variable Stars, 4, 222 (1933).

[2] P. P. Parenago, Variable Stars, 9, 89 (1953).

[3] A. H. Joy, $A p . \mathcal{F} .102$, I68 (1945).

[4] C. Hoffmeister, A.N. 278, 24 (1949).

[5] A. H. Joy, Publ. A.S.P. 58, 244 (1946).

[6] A. H. Joy, $A p$. . . 110, 424 (1949).

[7] P. N. Kholopov, Variable Stars, 8, 83 (1951).

[8] O. Struve, M. Rudkjøbing, Ap. F. 109, 92 (1949).

[9] G. Haro and A. Moreno, Bol. Obs. Tonantzintla y Tacubaya, No. 7, I I (1953).

[10] G. H. Herbig, Publ. A.S.P. 62, 142 (1950).

[II] G. Haro, A.J. 55, 72 (1950).

[12] G. H. Herbig, Ap.F. 111, I 5 (1950).

[13] G. H. Herbig, Four. R.A.S. Canada, 46, 222 (1952).

[14] G. Haro, $A p . \mathcal{F} .117,73$ (1953).

[15] G. H. Herbig, Ap.J. 119, 483 (1954).

[16] P. P. Parenago, Publ. Sternberg Astronomical Institute, 25, 225 (1954).

[17] G. H. Herbig, Trans. I.A.U. 8, 805 (1954).

[18] V. A. Ambartsumian, Bull. de l'Acad. de Sci. de l'U.R.S.S., sér. phys. 14, 5 (1950).

[19] P. N. Kholopov, Transactions of the Fourth Conference on Problems of Cosmogony, Moscow, 1955 .

[20] J. L. Greenstein, Publ. A.S.P. 62, 156 (1950).

[21] G. Haro, B. Iriarte and E. Chavira, Bol. Obs. Tonantzintla y Tacubaya, No. 8, 3 (1953).

[22] V. A. Ambartsumian, Comm. Burakan Obs. No. I3 (1954).

[23] I. M. Gordon, Comptes rendus de l'Acad. de Sci. de l'U.R.S.S., nouvelle sér. 97, 62 I (1954).

[24] L. E. Gurevitch, Transactions of the Second Conference on Problems of Cosmogony, p. 235, Moscow, 1953.

[25] R. F. Sanford, Publ. A.S.P. 59, i 34 (1947).

[26] V. A. Ambartsumian, Evolution of Stars and Astrophysics, Erevan, 1947.

[27] G. Haro and W. W. Morgan, Ap.J. 118, i6 (1953). 